\title{
INTERVENÇÃO EDUCATIVA NO LOCAL DE TRABALHO: PROMOÇÃO DA SAÚDE E PREVENÇÃO CARDIOVASCULAR COM HOMENS METALÚRGICOS
}

\author{
EDUCATIONAL INTERVENTION IN THE WORKPLACE: \\ CARDIOVASCULAR HEALTH PROMOTION AND \\ PREVENTION WITH METALLURGICAL MEN
}

\section{INTERVENCIÓN EDUCATIVA EN EL LUGAR DE TRABAJO: PROMOCIÓN DE LA SALUD Y PREVENCIÓN CARDIOVASCULAR CON HOMBRES METALÚRGICOS}

\author{
Sonia Silva Marcon ${ }^{1}$ \\ Gabriela Schiavon Ganassin ${ }^{2}$ \\ Guilherme Oliveira de Arruda ${ }^{3}$ \\ Elen Ferraz Teston ${ }^{4}$ \\ Mayckel da Silva Barreto 5 \\ Verônica Francisqueti Marquete \\ Patrícia Chatalov Ferreira
}

Como citar este artigo: Marcon SS, Ganassin GS, Arruda GO, Teston EF, Barreto MS, Marquete VF, et al. Intervenção educativa no local de trabalho: promoção da saúde e prevenção cardiovascular com homens metalúrgicos. Rev baiana enferm. 2021;35:e38619.

\begin{abstract}
Objetivo: analisar os efeitos de intervenção educativa nos fatores de risco cardiovasculares de homens metalúrgicos. Método: estudo de intervenção, com avaliação antes e depois, realizado com trabalhadores de indústria metalúrgica no interior do Paraná. Os dados foram coletados entre março e junho de 2014 em encontros semanais, no horário de descanso do almoço, seguindo os moldes do grupo operativo. O estudo foi iniciado com 135 homens (67 no grupo intervenção e 68 no controle) e concluído com 72 (35 e 37 respectivamente). Resultados: entre os participantes do grupo intervenção foi observado diminuição significativa de comportamentos de risco para saúde cardiovascular (sedentarismo, consumo excessivo de álcool, uso de temperos industrializados e hábitos alimentares inadequados) e redução da pressão arterial diastólica e do peso. Conclusão: intervenções no ambiente de trabalho constituem importante estratégia para o alcance do público masculino, além de serem efetivas na promoção de mudanças relacionadas ao estilo de vida.
\end{abstract}

Descritores: Estilo de Vida Saudável. Enfermagem. Educação em Saúde. Doenças Cardiovasculares. Fatores de Risco.

\footnotetext{
Enfermeira. Doutora em Filosofia da Enfermagem. Professora da Graduação e Pós-Graduação em Enfermagem da Universidade Estadual de Maringá. Maringá, Paraná, Brasil. soniasilva.marcon@gmail.com. http://orcid.org/0000-0002-6607-362X.

Enfermeira. Mestre em Enfermagem. Enfermeira do Tower Health Chestnut Hill Hospital. Philadelphia, Pensilvânia, Estados Unidos. http://orcid.org/0000-00021345-5026.

3 Enfermeiro. Doutor em Enfermagem. Professor da Graduação e Pós-Graduação em Enfermagem na Universidade Federal de Mato Grosso do Sul. Campo Grande, Mato Grosso do Sul, Brasil. http://orcid.org/0000-0003-1690-4808.

Enfermeira. Doutora em Enfermagem. Professora da Graduação e Pós-Graduação em Enfermagem na Universidade Federal de Mato Grosso do Sul. Campo Grande, Mato Grosso do Sul, Brasil. http://orcid.org/0000-000 I-6835-0574.

Enfermeiro. Doutor em Enfermagem. Coordenador do Curso de Enfermagem da Faculdade de Filosofia, Ciências e Letras de Mandaguari. Mandaguari, Paraná, Brasil. http://orcid.org/0000-0003-2290-84I8.

Enfermeira. Mestre em Enfermagem. Universidade Estadual de Maringá. Maringá, Paraná, Brasil. http://orcid.org/0000-0002-8070-609। .

Enfermeira. Especialista em Enfermagem nas Emergências. Universidade Estadual de Maringá. Maringá, Paraná, Brasil. http://orcid.org/0000-000 I-9409-5888.
} 
Objective: to analyze the effects of educational intervention on cardiovascular risk factors of metallurgical men. Method: intervention study, with evaluation before and after, carried out with workers of metallurgical industry in inland Paraná. Data were collected between March and June 2014 in weekly meetings, during lunch break time, following the operative group's molds. The study was started with 135 men (67 in the intervention group and 68 in the control group) and concluded with 72 (35 and 37, respectively). Results: among the participants of the intervention group, a significant decrease in risk behaviors for cardiovascular bealth (sedentary lifestyle, excessive alcohol consumption, use of industrialized spices and inadequate eating habits) and reduction of diastolic blood pressure and weight were observed. Conclusion: interventions in the work environment are an important strategy for reaching the male audience, besides being effective in promoting lifestyle-related changes.

Descriptors: Healthy Lifestyle. Nursing. Health Education. Cardiovascular Disease. Risk Factors.

Objetivo: analizar los efectos de la intervención educativa en los factores de riesgo cardiovascular de los hombres metalúrgicos. Método: estudio de intervención, con evaluación antes y después, realizado con trabajadores de la industria metalúrgica en el interior de Paraná. Los datos fueron recogidos entre marzo y junio de 2014 en reuniones semanales, durante la hora de descanso del almuerzo, de acuerdo con los moldes del grupo operativo. El estudio se inició con 135 hombres (67 en el grupo de intervención y 68 en el grupo de control) y concluyó con 72 (35 y 37, respectivamente). Resultados: entre los participantes del grupo de intervención, se observó una disminución significativa en las conductas de riesgo para la salud cardiovascular (estilo de vida sedentario, consumo excesivo de alcohol, uso de condimentos industrializados y hábitos alimenticios inadecuados) y reducción de la presión arterial diastólica y el peso. Conclusión: las intervenciones en el entorno de trabajo son una estrategia importante para llegar al público masculino, además de ser eficaces en la promoción de cambios relacionados con el estilo de vida.

Descriptores: Estilo de Vida Saludable. Enfermería. Educación para la Salud. Enfermedad Cardiovascular. Factores de Riesgo.

\section{Introdução}

Desde 2005, as doenças cardiovasculares (DCV) são as principais causas de morte no mundo e no Brasil e são representadas principalmente pela doença isquêmica do coração ${ }^{(1)}$. No ano de 2016, cerca de 17,9 milhões de pessoas no mundo morreram por DCV (31\% de todas as mortes globais). Cabe destacar que mais de 75\% das mortes por esta causa ocorrem em países de baixa e média renda ${ }^{(2)}$. O relatório "Estatística Cardiovascular - Brasil 2020" apresenta um panorama geral, com dados nacionais abrangentes sobre doença cardíaca e acidente vascular cerebral, em que se observa que a doença tem padrão semelhante em todas as regiões do país ${ }^{(3)}$. Especificamente no estado do Paraná, em 2015, as DCV ocuparam o primeiro lugar das causas de óbito e acarretaram 95.763 internações, com taxa de mortalidade de 182,4 /100.000 habitantes ${ }^{(1)}$.

Estudos apontam associação das DCV com o aumento da mortalidade em trabalhadores, em especial pela maior exposição a fatores ambientais e estressores, quando comparados com a po-

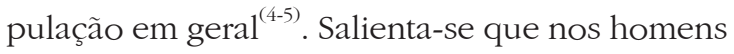
a pressão arterial sistólica elevada constitui o segundo maior contribuinte para morbimortalidade e incapacidade, seguida de riscos dietéticos e Índice de Massa Corpórea (IMC) elevado ${ }^{(6)}$.

No Brasil, a população masculina se destaca por adotar hábitos pouco saudáveis, como o tabagismo, dietas com baixo consumo de frutas e hortaliças, maior consumo de refrigerantes, de alimentos ultra processados, acentuado consumo de bebidas alcoólicas, além de prevalecer o excesso de peso ${ }^{(7)}$. Vale ressaltar que esses fatores contribuem para o aumento da morbimortalidade prematura em homens ${ }^{(4)}$, o que constitui uma realidade em diferentes regiões do país e do mundo, além de ser um problema de saúde pública $^{(8)}$.

No que concerne à promoção da saúde e prevenção de doenças, destaca-se que ações educativas voltadas especificamente para a população masculina têm se mostrado eficientes ${ }^{(9-10)}$, resultando inclusive no aumento da procura pelos serviços da Atenção Primária ${ }^{(11)}$. Essas ações, portanto, são de grande valia, em especial quando utilizam estratégias diferenciadas para abordar fatores e comportamentos de risco, 
que em sua maioria são modificáveis. Entretanto, a implementação dessas ações junto à população masculina constitui um desafio ${ }^{(12)}$, pois além de adotarem comportamentos pouco saudáveis ${ }^{(6,13-14)}$, procuram muito pouco os serviços de saúde da Atenção Primária e ainda existe o estereótipo estabelecido pela sociedade de que são seres fortes e invulneráveis ${ }^{(8)}$. Esses entraves favorecem o aparecimento de DCV no sexo masculino $^{(13)}$, e constituem uma das principais causas de morte nesse grupo.

Portanto, ainda que as intervenções de saúde necessitem ser organizadas de forma que sejam clinicamente mais apropriadas e mais efetivas do ponto de vista financeiro ${ }^{(14)}$, elas são essenciais para promover e manter mudanças nos hábitos de vida dos indivíduos ${ }^{(9)}$, e podem, inclusive, ser ampliadas para além do olhar biomédico, pois permitem a abordagem crítico-participativa na busca do autocuidado e empoderamento do sujeito $^{(9,15-16)}$. Nesse sentido, destaca-se o desafio para criar abordagens de intervenção acessíveis que levem a mudanças sustentáveis no estilo de vida, sobretudo de homens, e em especial, que considerem as particularidades dessa população em relação à procura e ao acesso aos serviços de saúde ${ }^{(9)}$.

Destaca-se que no cenário nacional, a saúde do homem tem constituído foco de interesse de profissionais e pesquisadores da área da saúde, os quais paulatinamente têm envidado esforços para o enfrentamento dos problemas envolvidos e identificação de abordagens específicas e mais efetivas na aproximação com essa população, em diferentes momentos e contextos. Importante destacar que este desafio ganha relevo especial no cenário atual da pandemia da COVID-19.

Estudos de intervenção voltados para a prevenção de DCV abordando trabalhadores ou a população de forma geral ainda são pouco frequentes no cenário brasileiro, especialmente entre operários de indústrias. Vale destacar que este público constitui uma classe específica de trabalhadores, que muitas vezes são sujeitos a uma rotina diferenciada de trabalho ${ }^{(17)}$. Ademais, entre os homens, é comum haver a priorização do trabalho em detrimento de ações de prevenção/promoção da saúde. Isso, por sua vez, atua como elemento determinante para a não adesão ao autocuidado e para a ocorrência de fatores de risco para o adoecimento ${ }^{(18)}$. Nesse contexto, implementar e avaliar intervenções educativas no espaço de trabalho pode possibilitar a proposição de ações oportunas e efetivas para a referida população.

Assim, o objetivo deste estudo foi analisar efeitos de intervenção educativa nos fatores de risco cardiovasculares de homens trabalhadores metalúrgicos.

\section{Método}

Estudo de intervenção realizado na matriz de uma empresa metalomecânica localizada na região metropolitana do município de Maringá, Paraná, que possui três filiais nos estados de São Paulo, Mato Grosso do Sul e Goiás. A escolha da empresa foi por conveniência devido a relações pessoais da pesquisadora que facilitaram $\mathrm{O}$ acesso e o desenvolvimento do estudo.

A empresa em questão iniciou suas atividades no ano de 1986 e atua na fabricação de equipamentos para o setor sucroalcooleiro, guindastes veiculares, equipamentos rodoviários e para movimentação de cargas. Ressalta-se que durante o estudo muitos trabalhadores foram desligados da empresa, visto que suas atividades estão relacionadas com a safra agrícola.

Participaram do estudo trabalhadores do sexo masculino que atenderam aos seguintes critérios de inclusão: trabalhar na empresa no período do início da coleta de dados, independente de tempo de atuação, e ter idade igual ou superior a 18 anos. Foram excluídos os trabalhadores afastados por licença médica ou férias.

De acordo com a listagem fornecida pela empresa, 230 trabalhadores do sexo masculino atendiam aos critérios de inclusão e exclusão e todos participaram da primeira etapa do estudo que tinha como propósito verificar o conhecimento sobre o risco de DCV. Nessa ocasião foram informados de que haveria uma segunda etapa do estudo e que, os que fossem sorteados para essa fase, receberiam um comunicado em 
seu cartão ponto informando o dia e a hora que deveriam comparecer na sala utilizada para a coleta de dados.

Para definir o número de trabalhadores que participaria da intervenção, considerou-se o total dos elegíveis (230), erro de estimativa de 1\%, confiabilidade e precisão da amostra de 95\%, e prevalência esperada de 50\%, resultando em 135 indivíduos, que foram selecionados aleatoriamente em dois estágios no programa Microsoft Excel ${ }^{\circledR}$. No primeiro estágio sorteou-se quem faria parte do estudo e, no segundo, quem seria alocado no grupo intervenção $(n=67)$ e no grupo controle ( $n=68$ ). Para tanto, a relação com os nomes dos 230 trabalhadores foi colocada em ordem alfabética e atribuídos números sequenciais. O mesmo processo ocorreu com os 135 sorteados, sendo realizado a randomização simples, com razão entre os grupos de 1:1, ficando 67 participantes no grupo intervenção $(n=67)$ e 68 no grupo controle (Figura 1) ${ }^{(19)}$. Todos os indivíduos sorteados concordaram em participar do estudo, não havendo necessidade de substituições para a composição dos grupos. Ressalta-se que após a randomização e as intervenções serem atribuídas a cada grupo, os pesquisadores e os participantes conheciam a composição e a definição dos grupos experimentais.

Figura 1 - Fluxograma de seleção dos participantes

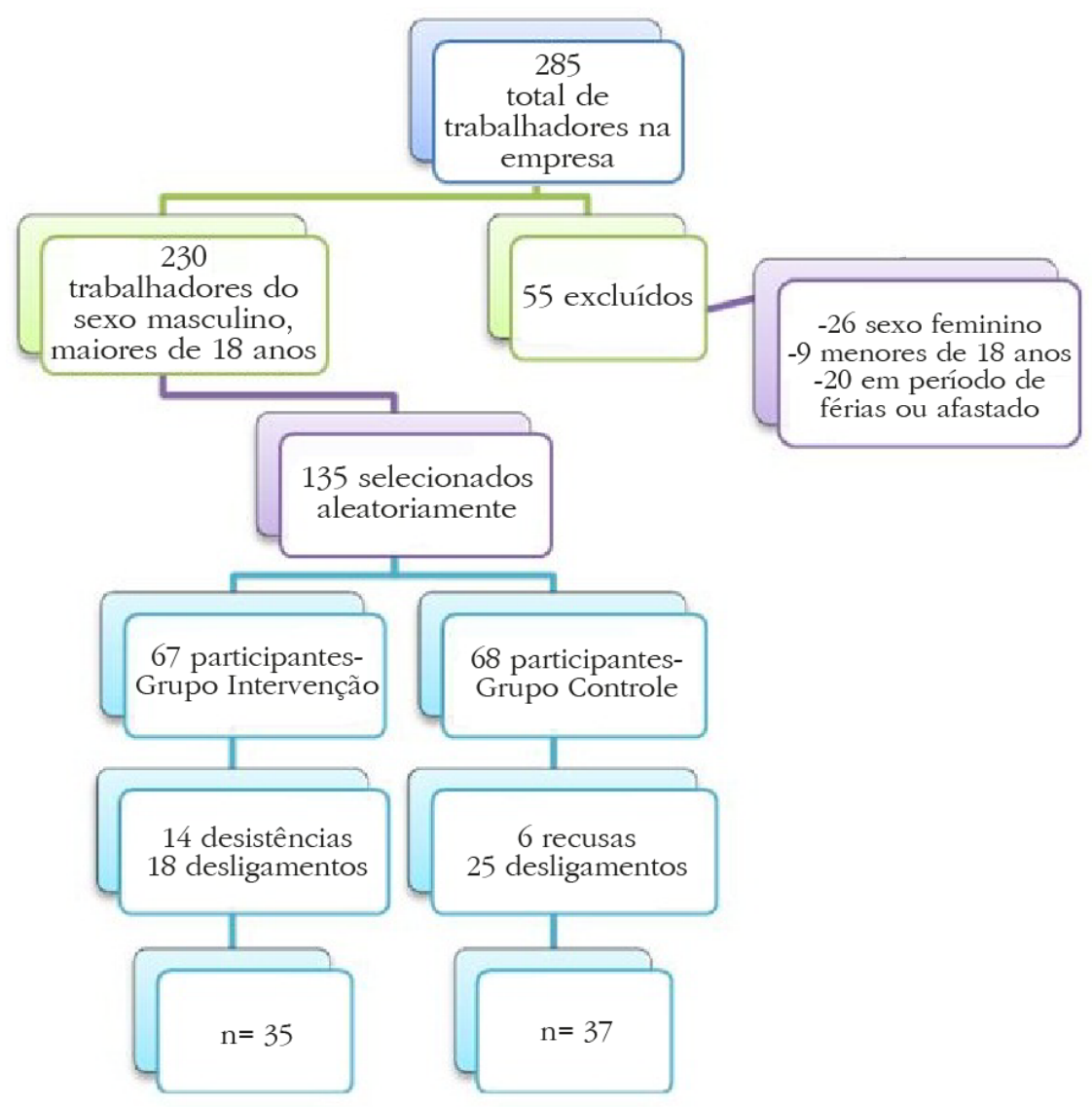

Fonte: Elaboração própria.

Os dados foram coletados no período de março a junho de 2014 em dois momentos antes e após a intervenção educativa -, mediante aplicação de instrumento semiestruturado e verificação de dados antropométricos. O instrumento utilizado abordava características sociodemográficas, antropométricas, clínicas (glicemia capilar, pressão arterial sistólica (PAS) e 
diastólica (PAD), e questões sobre Fatores de risco Cardiovascular, tabagismo, consumo excessivo de álcool, hábitos alimentares e prática de atividade física, extraídas de instrumentos validados, por permitirem avaliar o comportamento em saúde ${ }^{(7,13)}$.

Todos os trabalhadores alocados no grupo intervenção foram convidados a participar de um programa de educação para saúde, desenvolvido nos moldes dos grupos operativos ${ }^{(20)}$, tendo como referencial teórico o Modelo de Atenção às Condições Crônicas (MACC), que propõe a realização de ações de cuidado direcionadas à população em geral, aos indivíduos com comportamentos e fatores de risco, bem como aqueles que já possuem doença pré-existente, com vistas à redução de complicações ${ }^{(21)}$.

Foram adotados de forma mais específica os pressupostos relacionados aos níveis 1 e 2 do MACC. O nível 1 destina-se à população em geral e contempla ações de promoção da saúde, mediante a implementação de projetos intersetoriais com foco nos determinantes sociais da saúde, tais como condições de vida e trabalho e acesso a serviços. O nível 2 incorpora a prevenção das condições de saúde em subpopulações específicas, mediante a realização de intervenções sobre os determinantes sociais da saúde, ou seja, fatores de risco modificáveis aqueles relacionadas aos comportamentos e estilo de vida. São eles: dieta inadequada, excesso de peso, inatividade física, tabagismo e uso excessivo de álcool ${ }^{(21)}$.

Os indivíduos do grupo intervenção foram subdivididos em quatro subgrupos, pois, de acordo com os princípios do grupo operativo ${ }^{(20)}$, o número de participantes deve garantir a visualização de todos e, ao mesmo tempo, permitir que todos se manifestem. Durante três meses, os grupos reuniram-se semanalmente. Os encontros tiveram duração de aproximadamente 50 minutos e foram realizados logo após o almoço, durante o período de descanso, em sala cedida pela empresa. Foram conduzidos, gravados e mediados pela própria pesquisadora, auxiliada por uma enfermeira, que participou como observadora, registrando comportamentos não verbais e ajudando na verificação da pressão arterial, peso e glicemia capilar realizada no início e/ou ao final de cada encontro ${ }^{(19)}$.

Os encontros envolviam três momentos: acolhimento - verificação de sinais vitais e roda de conversa com espaço para o relato e troca de experiências, com foco nas dificuldades e conquistas da última semana; abordagem do tema do dia mediante exposição dialogada, precedida do levantamento dos conhecimentos prévios; fechamento - com esclarecimento de dúvidas e definição conjunta de tarefas a serem realizadas durante a semana e das metas a serem alcançadas pelo grupo em sua totalidade ou por alguns participantes.

Os temas abordados foram definidos previamente e em conjunto com os participantes no primeiro encontro, e incluíram: hipertensão arterial, diabetes, acidente vascular cerebral, infarto, câncer de próstata, alimentação saudável, atividade física, postura no trabalho, colesterol, tabagismo e consumo de bebida alcoólica, sobrepeso e obesidade ${ }^{(19)}$. Ao longo dos três meses, alguns profissionais de saúde foram convidados pela pesquisadora para abordarem aspectos específicos de sua área de atuação nos quatro subgrupos da intervenção. Foram eles: nutricionista, fisioterapeuta, endocrinologista, educador físico e enfermeiros.

Os 68 trabalhadores integrantes do grupo controle tiveram a pressão arterial aferida sempre que solicitado e a pesquisadora principal assumiu o compromisso de realizar atividades educativas após o término da coleta de dados, o que ocorreu em agosto e setembro do mesmo ano, mediante a realização de três palestras abertas a todos os trabalhadores, inclusive os do sexo feminino.

A avaliação pós-intervenção foi realizada uma semana após o término das atividades educativas, quando foram novamente aplicados todos os instrumentos utilizados na primeira etapa. Entretanto, para este estudo só foram consideradas as variáveis relacionadas aos fatores/comportamentos de risco cardiovascular, dicotomizadas em risco normal ou risco aumentado, segundo padrões da pesquisa do Sistema de Vigilância de 
Fatores de Risco e Proteção para Doenças Crônicas por Inquérito Telefônico (Vigitel) ${ }^{(3)}$.

O risco aumentado foi identificado quando ocorria:

a) consumo abusivo de bebida alcoólica ( sim/não) - cinco ou mais doses em uma mesma ocasião (uma dose de bebida destilada, uma lata de cerveja ou uma taça de vinho);

b) hábito atual de fumar ( $\operatorname{sim} /$ não), independente do número de cigarros por dia;

c) não realização de qualquer tipo de atividade física por pelo menos 50 minutos três ou mais vezes na semana, que somadas chegam a um tempo igual ou maior a 150 minutos de atividade física durante a semana ( $\operatorname{sim} /$ não);

d) consumo alimentar inadequado quando eram ingeridas frituras, bolos, bolachas e doces quatro ou mais dias na semana ou quando eram ingeridas frutas e verduras quatro ou menos dias na semana.

Para avaliação da pressão arterial, aferida após três a cinco minutos de repouso, utilizando método-padrão, considerou-se: PAS normal <130 mmHg, limítrofe de 130-139 mmHg e elevada $\geq 140 \mathrm{mmHg}$; PAD normal $<85 \mathrm{mmHg}$, limítrofe de 85-89 mmHg e elevada $\geq 90 \mathrm{mmHg}$. Entretanto, para fins estatísticos, os valores identificados como limítrofe ou elevado foram classificados como elevados.

Outros parâmetros considerados normais foram: glicemia capilar pós-prandial - mensurada com glicosímetro G-Tech Free ${ }^{\circledR}$ uma hora após o almoço - quando $\leq 160 \mathrm{mg} / \mathrm{dl}$; Porcentagem de Gordura Corporal (PCG) obtida com base no bioimpedanciômetro (BIA) InBody $\mathrm{R} 20^{\circledR} \leq 19 \%$; Índice de Massa Corporal (IMC) $\leq 25 \mathrm{~kg} / \mathrm{m}^{2}$ $\mathrm{mg} / \mathrm{dl}$ e Circunferência abdominal (CA) $\leq 92 \mathrm{~cm}$.

Os dados foram tabulados com dupla entrada em planilhas do Microsoft Excel ${ }^{\circledR}$ 2010, e posteriormente analisados pelo ambiente estatístico $\mathrm{R}$, versão 3.0.1. Procedeu-se análise descritiva e de normalidade dos dados conforme o teste de Shapiro-Wilk. Nos casos em que os parâmetros de normalidade foram satisfatórios, procedeu-se análise do teste t de Student para duas amostras dependentes quando comparadas duas médias (antes e depois) de um mesmo grupo, e o teste de $t$ de Student para duas amostras independentes, quando comparando as médias para dois grupos diferentes no momento basal e seguimento. Quando as variáveis tinham distribuição assimétrica, foi utilizado o teste de Wilcoxon na avaliação pareada e o teste de Mann-Whitney na avaliação independente. As comparações de proporções foram feitas com os testes de McNemar. Adotou-se nível de significância de 5\% para todos os testes ${ }^{(19)}$.

Destaca-se que no momento basal, os participantes dos dois grupos não apresentaram diferenças significativas em relação às características gerais (idade, estado civil, escolaridade e cor da pele) e no escore de conhecimento sobre fatores de risco para DCV, o que permitiu a comparabilidade entre eles.

O estudo foi desenvolvido em conformidade com a Resolução n. 466/12, do Conselho Nacional de Saúde (CNS), e aprovado pelo Comitê Permanente em Pesquisa com Seres Humanos da instituição signatária (Parecer n. 500.375/2013). Todos os participantes assinaram o Termo de Consentimento Livre e Esclarecido (TCLE) em duas vias.

\section{Resultados}

Os homens participantes deste estudo, em sua maioria, possuíam oito ou mais anos de estudo $(61,7 \%)$, tinham idade média de 38,23 $( \pm 12,44)$ anos, sendo predominantemente afrodescendentes $(58,7 \%)$, católicos (64,3\%) e com companheira (72,1\%). Em relação aos fatores de risco cardiovascular observou-se que 18,3\% tinham Hipertensão Arterial, 23\% eram tabagistas, 67\% não praticavam atividade física regularmente, $43,5 \%$ consumiam bebidas alcoólicas em excesso e $69,5 \%$ tinham dieta alimentar inadequada.

Dos 67 indivíduos incluídos no grupo intervenção, 35 (52,2\%) participaram da segunda avaliação, visto que 18 trabalhadores foram desligados da empresa antes do término da intervenção e 14 desistiram de participar das atividades educativas, justificado pelo fato de serem realizadas no horário de descanso. Vale salientar 
que os participantes demonstravam interesse pelas atividades que estavam sendo realizadas, visto que normalmente chegavam antes do horário agendado e traziam para o grupo dúvidas próprias e também de suas esposas. Inclusive, um dos participantes, mesmo estando de licença médica por fratura de antebraço, continuou frequentando os encontros. Para além, os colegas não integrantes dos subgrupos de intervenção, por diversas vezes procuravam as pesquisadoras para verificação da pressão arterial e perguntavam se podiam ficar para a reunião.

Dos 68 homens incluídos no grupo controle, 37 (54,4\%) participaram da segunda avaliação, visto que 6 recusaram responder novamente o questionário e 25 foram desligados da empresa. Ressalta-se que o número de trabalhadores desligados é decorrente do tipo de atividade da empresa, que é vinculada ao período da safra agrícola na região.
Na Tabela 1 estão representadas as características gerais dos grupos, assim como os fatores de risco relacionados ao comportamento em saúde cardiovascular. Observa-se que no grupo intervenção houve redução de quase $38 \%$ no índice de sedentarismo e de $31 \%$ e $32 \%$ no consumo excessivo de álcool e alimentação inadequada, respectivamente, e aumento de 20,9\% no deslocamento para o trabalho a pé ou de bicicleta. No grupo controle houve redução de aproximadamente $15 \%$ no índice de sedentarismo e de $22 \%$ na alimentação inadequada, porém houve uma redução de 10\% no deslocamento dos trabalhadores para o serviço a pé ou de bicicleta. Cabe salientar que durante a intervenção um dos participantes parou de fumar, desencadeando redução importante na porcentagem referente à variável hábito de fumar.

Tabela 1- Características gerais de comportamento e fatores de risco cardiovascular envolvidos entre grupos controle e intervenção pré e pós-intervenção. Maringá, Paraná, Brasil - 2014. (N=72) (continua)

\begin{tabular}{|c|c|c|c|c|c|c|c|c|c|}
\hline \multirow[b]{2}{*}{ Variáveis } & \multirow{2}{*}{\begin{tabular}{c|} 
Caracteris- \\
ticas gerais \\
p-valor \\
\end{tabular}} & \multicolumn{4}{|c|}{ Grupo Controle $(n=37)$} & \multicolumn{4}{|c|}{ Grupo Intervenção $(n=35)$} \\
\hline & & Antes & Depois & $\neq \frac{\mathrm{de}}{\bar{x}}$ & $\mathrm{p}$-valor & Antes & Depois & $\neq \frac{\mathrm{de}}{\bar{x}}$ & p-valor \\
\hline \multicolumn{10}{|l|}{$\begin{array}{l}\text { Fatores de risco } \\
\text { sociais }\end{array}$} \\
\hline $\begin{array}{l}\text { Escolaridade: } \geq 8 \\
\text { anos de estudo (\%) }\end{array}$ & $0,436^{\ddagger}$ & \multicolumn{2}{|c|}{56,8} & \multicolumn{6}{|c|}{65,7} \\
\hline \multicolumn{10}{|l|}{$\begin{array}{l}\text { Fatores de risco } \\
\text { biológicos }\end{array}$} \\
\hline $\begin{array}{l}\text { Prevalência de } \\
\text { sobrepeso (\%) }\end{array}$ & $0,096^{\ddagger}$ & 48,6 & 37,8 & & $0,376^{\ddagger}$ & 48,6 & 48,6 & & $0,544^{\ddagger}$ \\
\hline $\begin{array}{l}\text { Prevalência de } \\
\text { obesidade (\%) }\end{array}$ & $0,501^{\ddagger}$ & 8,1 & 8,1 & & & 20,0 & 20,0 & & \\
\hline Perda de peso (Kg) & $0,046^{\mathrm{a}}$ & & & 21 & $0,341^{\ddagger}$ & & & 10 & $0,555^{\ddagger}$ \\
\hline PA Sistólica (mmHg) & $0,762^{\mathrm{a}}$ & 133,2 & 136,0 & & $0,132^{\dagger}$ & 130,6 & 128,6 & & $0,455^{\dagger}$ \\
\hline $\begin{array}{l}\text { PA Diastólica } \\
(\mathrm{mmHg})\end{array}$ & $0,653^{a}$ & 90,2 & 88,4 & & $0,386^{\dagger}$ & 87,7 & 79,7 & & $<0,001^{\dagger}$ \\
\hline \multicolumn{10}{|l|}{$\begin{array}{l}\text { Fatores de risco } \\
\text { comportamentais }\end{array}$} \\
\hline Inatividade física (\%) & $0,059^{\mathrm{a}}$ & 72,9 & 62,1 & & $1,000 \ddagger$ & 68,6 & 42,8 & & $0,344 \neq$ \\
\hline $\begin{array}{l}\text { Consumo excessivo } \\
\text { de álcool (\%) }\end{array}$ & $0,600^{a}$ & 43,2 & 43,2 & & $1,000 \neq$ & 35,1 & 24,3 & & $0,344 \neq$ \\
\hline Hábito de fumar (\%) & $0,118^{\mathrm{a}}$ & 29,7 & 29,7 & & $1,000 \ddagger$ & 13,5 & 10,8 & & $1,000 \neq$ \\
\hline $\begin{array}{l}\text { Deslocamento para } \\
\text { o trabalho: a pé ou } \\
\text { bicicleta }(\%)\end{array}$ & $0,345^{\mathrm{a}}$ & 54,0 & 48,6 & & $0,625 \neq$ & 42,5 & 51,4 & & $0,453 \neq$ \\
\hline $\begin{array}{l}\text { Alimentação } \\
\text { inadequada* }(\%)\end{array}$ & $0,098^{\mathrm{a}}$ & 62,2 & 48,6 & & $0,302 \ddagger$ & 75,7 & 51,4 & & $0,035 \ddagger$ \\
\hline
\end{tabular}


Tabela 1- Características gerais de comportamento e fatores de risco cardiovascular envolvidos entre grupos controle e intervenção pré e pós-intervenção. Maringá, Paraná, Brasil - 2014. (N=72) (conclusão)

\begin{tabular}{|c|c|c|c|c|c|c|c|c|c|}
\hline \multirow[b]{2}{*}{ Variáveis } & \multirow{2}{*}{$\begin{array}{c}\text { Caracteris- } \\
\text { ticas gerais } \\
\text { p-valor }\end{array}$} & \multicolumn{4}{|c|}{ Grupo Controle $(n=37)$} & \multicolumn{4}{|c|}{ Grupo Intervenção $(n=35)$} \\
\hline & & Antes & Depois & $\neq \frac{\mathrm{de}}{\bar{x}}$ & p-valor & Antes & Depois & $\neq \frac{\mathrm{de}}{\bar{x}}$ & p-valor \\
\hline \multicolumn{10}{|l|}{$\begin{array}{l}\text { Fatores de risco } \\
\text { comportamentais }\end{array}$} \\
\hline $\begin{array}{l}\text { Refeições diárias } \\
\text { (x/dia) }\end{array}$ & $0,659^{\ddagger}$ & $\begin{array}{c}2,97 \pm \\
1,14\end{array}$ & $\begin{array}{c}3,08 \pm \\
0,86\end{array}$ & 0,1 & $0,568^{\dagger}$ & $\begin{array}{c}3,08 \pm \\
1,01\end{array}$ & $\begin{array}{c}3,51 \pm \\
1,33\end{array}$ & 0,4 & $0,015^{\dagger}$ \\
\hline $\begin{array}{l}\text { Ingesta de verduras e } \\
\text { legumes (x/semana) }\end{array}$ & $0,919^{\mathrm{a}}$ & $\begin{array}{c}4,62 \pm \\
2,64\end{array}$ & $\begin{array}{c}1,94 \pm \\
2,27\end{array}$ & $-2,7$ & $0,117^{\dagger}$ & $\begin{array}{c}5,42 \pm \\
1,97\end{array}$ & $\begin{array}{c}5,34 \pm \\
1,71\end{array}$ & $-0,1$ & $0,924^{\dagger}$ \\
\hline $\begin{array}{l}\text { Ingesta de fritura } \\
(\mathrm{x} / \text { semana })\end{array}$ & $0,789^{\mathrm{a}}$ & $\begin{array}{c}2,72 \pm \\
2,31\end{array}$ & $\begin{array}{c}2,83 \pm \\
2,25\end{array}$ & 0,1 & $0,446^{\dagger}$ & $\begin{array}{l}3,14 \pm \\
2,48\end{array}$ & $\begin{array}{c}3,17 \pm \\
2,18\end{array}$ & 0,03 & $0,289^{\dagger}$ \\
\hline $\begin{array}{l}\text { Uso de temperos } \\
\text { industriais }(\mathrm{x} / \text { semana })\end{array}$ & $0,941^{\mathrm{a}}$ & $\begin{array}{c}5,37 \pm \\
2,73\end{array}$ & $\begin{array}{l}3,83 \pm \\
3,08\end{array}$ & $-1,5$ & $0,271^{\dagger}$ & $\begin{array}{c}3,54 \pm \\
3,11\end{array}$ & $\begin{array}{c}1,91 \pm \\
2,39\end{array}$ & $-1,6$ & $0,689^{\dagger}$ \\
\hline $\begin{array}{l}\text { Ingesta de água } \\
\text { (litros/dia) }\end{array}$ & $0,832^{\ddagger}$ & & & & $0,051^{\dagger}$ & & & & $0,005^{\dagger}$ \\
\hline
\end{tabular}

Fonte: Elaboração própria.

Notas: Dados apresentados como médias $(\bar{x})$ e desvios padrão, com exceção dos que estão indicados como porcentagens (\%).

* De acordo com o Ministério da Saúde ${ }^{(22)}$; † Teste de Wilcoxon; ‡ Teste t-Student; $\neq$ Teste McNemar; ${ }^{a}$ Teste de Mann-Whitney.

Quanto aos hábitos alimentares (Tabela 1), os fatores que se mostraram mais sensíveis à intervenção foram o número de refeições diárias e o consumo de água. Ademais, observou-se que a frequência de hábitos alimentares inadequados diminuiu aproximadamente 20\% em ambos os grupos. Contudo, a análise de correlação entre o momento inicial e final mostrou que no grupo intervenção as variáveis número de refeições diárias, consumo de água e alimentação inadequada apresentaram alteração significativa, enquanto que no grupo controle, isto ocorreu apenas para consumo de água.

Já em relação às variáveis antropométricas e hemodinâmicas, apenas a PAD apresentou redução significativa; isto ocorreu apenas no grupo intervenção. Em relação ao peso corporal, apesar de reduções importantes no âmbito individual, elas não foram suficientes para diminuir de forma significativa o peso médio do grupo.

\section{Discussão}

Os dados mostraram que mesmo com redução significativa na ingesta de água por parte dos metalúrgicos do grupo intervenção, houve aumento significativo de hábitos alimentares saudáveis (redução na porcentagem de homens que usavam temperos industrializados e aumento no número de refeições realizadas por dia). Além disso, embora sem diferença estatisticamente significativa, constatou-se o início de outros comportamentos que podem impactar na prevenção de doenças cardiovasculares como, por exemplo, redução da inatividade física, do consumo excessivo de álcool e do hábito de fumar. Estes resultados oferecem subsídios para o planejamento e a implementação de ações de educação para a saúde pelos enfermeiros e outros integrantes da equipe multiprofissional, em especial aqueles atuantes na Atenção Primária. Essas ações, conforme proposto pelo MACC ${ }^{(21)}$, devem priorizar a promoção da saúde e a prevenção de doenças e agravos, com foco nos fatores de risco modificáveis, mais especificamente aqueles relacionados com os hábitos de vida.

Estudo experimental não randomizado realizado no Brasil com 157 adultos de ambos os sexos identificou que as intervenções constituídas por educação em saúde sobre o exercício físico e aulas semanais de exercícios cardiorrespiratórios foram efetivas - aumentaram a prática de atividade física. Entretanto, a educação em saúde foi mais efetiva na manutenção dessa prática no período pós-intervenção. Nesse sentido, os autores recomendam que no âmbito do 
Sistema Único de Saúde (SUS), para promoção da atividade física, sejam utilizadas ambas as modalidades de intervenções ${ }^{(23)}$.

Em contrapartida, estudo de intervenção realizado com 54 homens residentes na zona rural dos Estados Unidos constatou que os participantes tinham ciência dos fatores de risco cardiometabólicos modificáveis (atividade física, dieta e uso de tabaco); contudo, não se sentiam capazes de adotar comportamentos saudáveis, exceto aqueles participantes que vivenciaram na família a ocorrência de uma emergência cardiovascular e os que estavam mais próximos dos sessenta anos. Assim, os autores concluíram que a ocorrência de uma emergência familiar e o desejo de mitigar os declínios cognitivos ocasionados pelo envelhecimento eram fatores que incentivavam a mudança de hábitos naqueles homens ${ }^{(24)}$. Desse modo, infere-se que nas atividades de educação em saúde, é importante fazer uso de exemplos práticos e de estratégias que estimulem os indivíduos a compartilharem experiências vivenciadas, pois isto pode favorecer a conscientização e mudanças, ainda que pequenas, nos hábitos/comportamentos diários.

No que concerne ao contexto específico de homens trabalhadores, destaca-se o desafio do desenvolvimento, implementação e adesão de ações com enfoque na promoção da saúde e de prevenção de doenças, que compõem dois eixos estratégias do MACC. Estudo realizado com homens trabalhadores rurais, cujo objetivo foi compreender as percepções frente às práticas de cuidado, apontou o predomínio da visão de cuidado centrada no modelo biomédico, além do fato de considerarem o trabalho como uma ferramenta para cuidar da saúde ${ }^{(25)}$. Por conseguinte, ações relacionadas à promoção da saúde e prevenção de doenças junto à população em geral, mais especialmente aos homens, necessitam alcançar a significação de saúde e doença e oportunizar espaços de discussões, em especial no próprio ambiente de trabalho.

Ademais, estudo brasileiro destacou as ações bem sucedidas promovidas pelas equipes da Estratégia Saúde da Família junto aos pacientes com diabetes, que incluíam desde a oferta de grupos (de apoio nutricional, caminhadas, educação em saúde), consultas individuais, distribuição de medicamentos, até o "bom atendimento". Isso demonstra a importância da qualidade da relação entre pacientes e profissionais de saúde, no empoderamento, participação e responsabilidade do paciente em relação ao seu plano de cuidados $^{(26)}$. Desse modo, ao adentrar os espaços extramuros das Unidades de Saúde, a equipe necessita inicialmente estabelecer relação de confiança com o público-alvo e fazer uso de estratégias que despertem o interesse pela discussão das temáticas de promoção e prevenção, como, por exemplo, a valorização da escuta ativa.

Os resultados deste estudo também corroboram os de um estudo quase experimental realizado com pessoas com Síndrome metabólica da China ${ }^{(27)}$, que identificou que um programa de intervenção no estilo de vida orientado pelo Modelo de Promoção da Saúde, liderado por enfermeiros, melhorou efetivamente a autoeficácia e a implementação de comportamentos promotores de saúde, o que inclui a saúde cardiovascular. Do mesmo modo, estudo piloto randomizado controlado, realizado com 59 homens nos Estados Unidos, identificou impacto positivo de uma intervenção com teleatendimento na atenção primária. O propósito era promover mudanças no estilo de vida para reduzir fatores de risco cardiovascular, e, ao final de 12 semanas, uma proporção significativamente maior de participantes do grupo intervenção perdeu pelo menos $5 \%$ do peso inicial ${ }^{(11)}$.

Pesquisa realizada na comunidade de Paraisópolis, São Paulo, com sete profissionais e com nove homens adultos, inferiu que as ações dialógicas realizadas por grupo focal contribuíram para a promoção da saúde dos participantes ao estimularem o (re)conhecimento de determinantes de suas condições de vida e saúde ${ }^{(11)}$. Ensaio clínico randomizado, desenvolvido com 72 usuários com Diabetes Mellitus de um município da Amazônia Brasileira, identificou que os círculos de cultura com abordagem educativa podem aperfeiçoar o autocuidado relacionado à alimentação saudável, à prática de exercício físico e cuidados com os pés, resultando em 
10

Intervenção educativa no local de trabalho: promoção da saúde e prevenção cardiovascular com homens metalúrgicos

melhora de alguns parâmetros de risco cardiovascular (glicemia capilar em jejum, pressão arterial sistólica e diastólica, Índice de Massa Corpórea e circunferência abdominal) ${ }^{(28)}$.

No presente estudo, além de ter sido observado diminuição da inatividade física, do consumo de bebidas alcoólicas e de alimentação inadequada, houve aumento na proporção de indivíduos que passaram a se deslocar para o trabalho a pé ou de bicicleta. Isso pode ter sido influenciado pelo incentivo dos profissionais atuantes no programa de educação em saúde, que fomentaram a adoção de alternativas capazes de modificar o cotidiano e que, ao mesmo tempo, caracterizam um estilo de vida mais saudável. Cabe destacar que o incentivo e a pactuação de pequenas mudanças no cotidiano constitui uma das estratégias do MACC $^{(21)}$.

Nesse tocante, estudo controlado randomizado, realizado com 1.113 torcedores do sexo masculino, que utilizou um programa Europeu de Fãs em Treinamento (EuroFIT) como intervenção para melhorar a atividade física e o tempo sedentário, constatou melhora nos hábitos alimentares, diminuição na ingestão de álcool, reduções no peso corporal médio, IMC, circunferência da cintura e de significativos riscos cardiovasculares ${ }^{(29)}$. Esses resultados levaram os autores a concluírem que todas as diferentes maneiras de promover e incentivar a promoção da saúde são plausíveis e que a atividade física constitui um meio efetivo para envolver os homens com questões relacionadas à saúde ${ }^{(29)}$. Nesse sentido, pensar em ações conjuntas com as empresas, visando possibilitar que atividades laborais possam ser implementadas na rotina do serviço, constitui um incentivo inicial à realização de exercício físico.

No que se refere aos hábitos alimentares, observou-se redução, embora não significativa, no número de indivíduos que faziam uso de temperos industrializados, o que possibilita afirmar que isso talvez tenha contribuído para a redução significativa nos níveis de PAD no grupo intervenção. Ademais, o aumento na média do "número de refeições diárias" também demonstra o esforço empreendido pelos trabalhadores para obterem um estilo de vida mais saudável. Esse resultado corrobora o de intervenção constituída por consultas tradicionais associadas a aconselhamento personalizado, mediante ligações telefônicas dos enfermeiros da atenção básica de Concepción, Chile. Nos homens, os efeitos da intervenção foram: diminuições na pressão sistólica e diastólica, circunferência abdominal, colesterol total, colesterol de lipoproteínas de baixa densidade, índice aterogênico, fator de risco cardiovascular e risco coronariano ${ }^{(30)}$.

Intervenção abordando pessoas com diabetes constatou que, dentre as atividades propostas relacionadas com o autocuidado, a adoção de alimentação saudável foi uma das que obtiveram menores índices de adesão, justificado pela questão cultural dos participantes ${ }^{(28)}$. Esses resultados confirmam que o compromisso individual dos participantes em relação à adoção de hábitos de vida mais saudável deve ser sempre incentivado e encorajado pelos profissionais de saúde, os quais devem inclusive esclarecer que elas nem sempre são capazes de, em pouco tempo, resultarem em mudanças significativas no peso corporal, sendo importante a persistência em longo prazo.

Outro aspecto que precisa ser ponderado é sobre o custo para que ocorram as mudanças no padrão alimentar dos homens. Estudo realizado com dados do Instituto Brasileiro de Geografia e Estatística (IBGE) identificou que, dos 55.406 domicílios investigados, 39,4\% foram classificados em Insegurança Alimentar, dos quais, a maioria (93,9\%) identificada pela "dificuldade de levar a vida até o fim do mês com a renda disponível". Ressalta-se que aspectos econômicos, condições de vida insatisfatórias, problemas no domicílio e do entorno apresentaram associação significativa com Insegurança Alimentar ${ }^{(31)}$. Destarte, o custo interfere no consumo de frutas, verduras e legumes, evidenciando que para a população brasileira em geral, incluindo os homens, questões relacionadas à renda impactam na seleção dos alimentos a serem consumidos. Essa realidade precisa ser considerada nas intervenções em saúde voltadas para a escolha de alimentos mais saudáveis. 
Por fim, é importante ressaltar que os resultados deste e de outros estudos apontam a relevância de intervenções, ainda que de curta duração, na mudança de hábitos alimentares. No caso de homens, isto parece ser particularmente importante quando ocorre no local de trabalho ${ }^{(12)}$. Estudos futuros, no entanto, precisam ser realizados, não só para identificar se estas mudanças são duradouras ou não, mas principalmente para identificar os seus benefícios.

As limitações deste estudo incluem o curto período de acompanhamento, o tamanho da amostra, decorrente principalmente das perdas ocorridas ao longo da intervenção. Ademais, uma dificuldade encontrada foi a necessidade de adequar os horários do pesquisador com os sujeitos da pesquisa, além da contaminação de informações que pode ter ocorrido entre os trabalhadores dos dois grupos, por trabalharem na mesma empresa e, às vezes, até nos mesmos setores, lado a lado, e inclusive compartilharem do mesmo ambiente durante o período de almoço.

\section{Conclusão}

A comparação antes e depois da intervenção mostrou diferenças estatisticamente significativas apenas para as variáveis: número de refeições diárias, alimentação inadequada, consumo de água diário e pressão arterial diastólica. Contudo, ainda assim, pode-se concluir que a intervenção educativa foi efetiva e capaz de produzir impacto positivo em trabalhadores do sexo masculino em relação aos fatores de risco cardiovascular, especialmente os relacionados ao comportamento - prática de atividade física e hábitos alimentares.

Ressalta-se que os resultados deste estudo demonstram que as práticas cuidativas com os homens trabalhadores metalúrgicos são eficazes e necessárias para a promoção da saúde e prevenção de agravos e complicações. Assim, destaca-se o papel que o profissional enfermeiro pode desempenhar nesse processo, como parte de uma de suas atribuições, a de educador em saúde. Mostra-se necessário que os enfermeiros sejam estimulados e incentivados a adotar intervenções em saúde direcionadas a essa população, a fim de possibilitar a redução da morbimortalidade nesse público específico.

A melhora no comportamento alimentar do grupo intervenção foi consistente com o de outros estudos e indica a efetividade de programas de intervenção voltados à educação em saúde, assim como demonstra a importância e a necessidade de mais estudos com trabalhadores, especialmente os do sexo masculino, visto as limitações que estes encontram para procurar os serviços de saúde no âmbito da atenção primária em saúde.

\section{Colaborações:}

1 - concepção, projeto, análise e interpretação dos dados: Sonia Silva Marcon, Gabriela Schiavon Ganassin e Guilherme Oliveira de Arruda;

2 - redação do artigo e revisão crítica relevante do conteúdo intelectual: Sonia Silva Marcon, Gabriela Schiavon Ganassim, Guilherme Oliveira de Arruda, Elen Ferraz Teston, Mayckel da Silva Barreto, Verônica Francisqueti Marquete e Patrícia Chatalov Ferreira;

3 - aprovação final da versão a ser publicada: Sonia Silva Marcon, Gabriela Schiavon Ganassim, Guilherme Oliveira de Arruda, Elen Ferraz Teston e Mayckel da Silva Barreto.

\section{Referências}

1. Paraná. Secretaria de Estado da Saúde do Paraná. Superintendência de Atenção à Saúde. Linha Guia de Hipertensão Arterial/ SAS. 2a ed. Curitiba: SESA; 2018.

2. World Health Organization. Cardiovascular diseases (CVDs) [Internet]. Geneva (CH); 2016 [cited 2016 Mar 29]. Available from: https:// www.who.int/news-room/fact-sheets/detail/ cardiovascular-diseases-(cvds)

3. Oliveira GMM, Brant LCC, Polanczyk CA, Biolo A, Nascimento BR, Malta DC, et al. Cardiovascular Statistics - Brazil 2020. Arq Bras Cardiol. 2020;115(3):308-439. DOI: http://dx.doi. org/10.36660/abc.20200812

4. Eriksson HP, Söderberg M, Neitzel RL, Torén K, Andersson E. Cardiovascular mortality in a Swedish 
cohort of female industrial workers exposed to noise and shift work. Int Arch Occup Environ Health. 2020. DOI: https://doi.org/10.1007/ s00420-020-01574-x

5. Tannis C, Chernov C, Perlman S, McKelvey W, Toprani A. Cardiovascular Health Risk Behaviors by Occupation in the NYC Labor Force. J Occup Environ Med. 2020;62(9):757-63. DOI: 10.1097/ JOM.0000000000001960

6. Souza MFM, Passos VMA, Malta D, França EB, Abreu D, Araújo VEM, et al. Burden of disease in Brazil, 1990-2016: a systematic subnational analysis for the Global Burden of Disease Study 2016. Lancet. 2018;392(10149):760-75. DOI: 10.1016/S0140-6736(18)31221-2

7. Brasil. Ministério da Saúde. Secretaria de Vigilância em Saúde. Departamento de Análise em Saúde e Vigilância de Doenças Não Transmissíveis. VIGITEL Brasil 2019: vigilância de fatores de risco e proteção para doenças crônicas por inquérito telefônico [Internet]. Brasília; 2020 [cited 2020 Jul 25]. Available from: https://portalarquivos2. saude.gov.br/images/pdf/2019/julho/25/vigitelbrasil-2018.pdf

8. Baker P, Shand T. Men's health: time for a new approach to policy and practice? J Glob Health. 2017;7(1):010306. DOI: 10.7189/ jogh.07.010306

9. Arruda GO, Marcon SS, Peruzzo HE, Ruiz AGB, Back IR, Nass EMA, et al. Intervenção educativa em homens com diabetes mellitus: efeitos sobre comportamentos e perfil antropométrico. Acta paul enferm. 2020;33:eAPE20190128. DOI: 10.37689/ acta-ape/2020ao0128

10. Torres VN, Willians EC, Ceballos RM, Donovan DM, Ornelas IJ. Participant Satisfaction and Acceptability of a Culturally Adapted Brief Intervention to Reduce Unhealthy Alcohol Use Among Latino Immigrant Men. Am J Mens Health. 2020;14(3):1557988320925652. DOI: $10.1177 / 1557988320925652$

11. Marra MV, Lilly CL, Nelson KR, Woofter DR, Malone J. A Pilot Randomized Controlled Trial of a Telenutrition Weight Loss Intervention in Middle-Aged and Older Men with Multiple Risk Factors for Cardiovascular Disease. Nutrients. 2019;11(2):229. DOI: 10.3390/nu11020229

12. Seaton CL, Bottorff JL, Jones-Bricker M, Lamont S. The Role of Positive Emotion and EgoResilience in Determining Men's Physical Activity
Following a Workplace Health Intervention. Am J Mens Health. 2018;12(6):1916-28. DOI: $10.1177 / 1557988318803744$

13. Précoma DB, Oliveira GMM, Simão AF, Dutra OP, Coelho OR, Izar MCO, et al. Updated Cardiovascular Prevention Guideline of the Brazilian Society of Cardiology - 2019. Arq Bras Cardiol. 2019;113(4):787-891. DOI:10.5935/ abc. 20190204

14. Anderson L, Brown JPR, Clark AM, Dalal H, Rossau HKK, Bridges C, et al. Patient education in the management of coronary heart disease. Cochrane Database Syst Rev. 2017;(6):CD008895. DOI: 10.1002/14651858.CD008895.pub3

15. Santos FNP, Baldissera VDA, Toledo RF. Conversa de Boteco: participação, educação e promoção da saúde do homem. Esc Anna Nery. 2019;23(3):e20190006. DOI: 10.1590/2177-9465-ean-2019-0006

16. Köhler AK, Tingström P, Jaarsma T, Nilsson S. Patient empowerment and general self-efficacy in patients with coronary heart disease: a crosssectional study. BMC Fam Pract. 2018;19(1):76. DOI: 10.1186/s12875-018-0749-y

17. Silveira PM, Silva KS, Mello GT, Knebel MTG, Borgatto AF, Nahas MV. Tabagismo em trabalhadores da indústria no Brasil: associação com fatores sociodemográficos, consumo de bebidas alcoólicas e nível de estresse. J bras pneumol. 2020;46(1):e20180385. DOI: 10.1590/1806-3713/ e20180385

18. Barros CT, Gontijo DT, Lyra J, Lima LS, Monteiro EMLM. "Mas se o homem cuidar da saúde fica meio paradoxal ao trabalho": relação entre masculinidades e cuidado à saúde para homens jovens em formação profissional. Saúde Soc. 2018;27(2):423-34. DOI: 10.1590/ S0104-12902018166057

19. Ganassin GS, Silva EM, Pimenta AM, Marcon SS. Efetividade da intervenção educativa no conhecimento de homens relacionado às doenças cardiovasculares. Acta paul enferm. 2016;29(1):38-46. DOI: https://doi.org/10.1590/ 1982-0194201600006

20. Pichon-Rivière E. Teoria do vínculo. São Paulo: Martins Fontes; 1988.

21. Mendes EV. O cuidado das condições crônicas na atenção primária à saúde: o imperativo da consolidação da estratégia da saúde da família. Brasília (DF): Organização Pan-Americana da 
Saúde; 2012 [cited 2016 Apr 14]. Available from: https://bvsms.saude.gov.br/bvs/publicacoes/ cuidado_condicoes_atencao_primaria_saude.pdf

22. Brasil. Ministério da Saúde. Secretaria de Atenção à Saúde. Guia alimentar para a população brasileira [Internet]. 2a ed. Brasília (DF); 2014 [cited 2014 Mar 2]. Available from: https://bvsms.saude.gov. br/bvs/publicacoes/guia_alimentar_populacao_ brasileira_2ed.pdf

23. Ribeiro EHC, Garcia LMT, Salvador EP, Costa EF, Andrade DR, Latorre MRDO, et al. Assessment of the effectiveness of physical activity interventions in the Brazilian Unified Health System. Rev Saúde Pública. 2017;51:56. DOI: 10.1590/S1518-8787.2017051006654

24. Morgan EH, Graham ML, Folta SC, Seguin RA. A qualitative study of factors related to cardiometabolic risk in rural men. BMC Public Health. 2016;16:305. DOI: 10.1186/ s12889-016-2977-1

25. Miranda SVC, Duraes OS, Vasconcellos LCF. A visão do homem trabalhador rural norte-mineiro sobre o cuidado em saúde no contexto da atenção primária à saúde. Ciênc saúde coletiva. 2020;25(4):1519-28. DOI: 10.1590/1413-81232020254.21602018

26. Moreschi C, Rempel C, Backes DS, Pombo CNF, Siqueira DF, Pissaia LF. <b>Ações das equipes da ESF para a qualidade de vida das pessoas com diabetes. Ciênc Cuid Saúde. 2018;17(2):e-41000. DOI: 10.4025/cienccuidsaude.v17i2.41000

27. Zheng X, Yu H, Qiu X, Chair SY, Wong EML, Wang Q. The effects of a nurse-led lifestyle intervention program on cardiovascular risk, self-efficacy and health promoting behaviours among patients with metabolic syndrome: Randomized controlled trial. Int J Nurs Stud. 2020;109:103638. DOI: 10.1016/j.ijnurstu.2020. 103638

28. Correa ST, Castelo-Branco S. Amandaba no Caeté: ćŕrculos de cultura como prática educativa no autocuidado de portadores de diabetes. Saúde debate. 2019;43(123):1106-19. DOI: 10.1590/0103-1104201912310

29. Wyke S, Bunn C, Andersen E, Silva MN, van Nassau F, McSkimming $\mathrm{P}$, et al. The effect of a programme to improve men's sedentary time and physical activity: The European Fans in Training (EuroFIT) randomised controlled trial. PLoS Med. 2019;16(2):e1002772. DOI: https://doi. org/ 10.1371/journal.pmed.1002772

30. Vilchez Barboza V, Klijn TP, Salazar Molina A, Sáez Carrillo KL. Eficácia de uma intervenção personalizada de aconselhamento em enfermagem, presencial e telefônica, para fatores de risco cardiovascular: ensaio clínico controlado. Rev Latino-Am Enfermagem. 2016;24:e2747. DOI: $10.1590 / 1518-8345.0626 .2747$

31. Araújo ML, Nascimento DR, Lopes MS, Passos CM, Lopes ACS. Condições de vida de famílias brasileiras: estimativa da insegurança alimentar. Rev bras estud popul. 2020;37:e0110. DOI: $10.20947 / \mathrm{s} 0102-3098 \mathrm{a} 0110$

Recebido: 4 de setembro de 2020

Aprovado: 7 de novembro de 2020

Publicado: 19 de janeiro de 2021

A Revista Baiana de Enfermagem utiliza a Licença Creative Commons - Atribuição-NãoComercial 4.0 Internacional. https://creativecommons.org/licenses/by-nc/4.0/

Este artigo é de acesso aberto distribuído sob os termos da Licença Creative Commons (CC BY-NC). Esta licença permite que outros remixem, adaptem e criem a partir do seu trabalho para fins não comerciais. Embora os novos trabalhos tenham de lhe atribuir o devido crédito e não possam ser usados para fins comerciais, os usuários não têm de licenciar esses trabalhos derivados sob os mesmos termos. 